\title{
DEMÊNCIA FRONTOTEMPORAL SUBDIAGNOSTICADA
}

Senhor Editor - No artigo intitulado Prevalence of presenile dementia in a tertiary outpatient clinic ${ }^{1}$, os autores reconhecem a baixa prevalência de demência frontotemporal (DFT) encontrada no estudo, porém não conseguiram explicar tal achado, afirmando apenas que provavelmente não se tratava de falhas no procedimento diagnóstico adotado. Acrescentam ainda que talvez a baixa prevalência encontrada para a DFT siga a prevalência geral desta forma de demência no Brasil, não obstante não existirem dados epidemiológicos para tal afirmação. De fato, vários são os trabalhos que apontam para uma prevalência mais representativa desta forma de demência no universo das demências pré-senis. Um importante estudo de revisão coloca a DFT como a terceira causa mais importante de demência no grupo de pacientes abaixo de 65 anos, com uma taxa de aproximadamente a metade daquela encontrada para a doença de Alzheimer - DA (que seria a causa mais comum de demência pré-senil) e similar à encontrada para demência vascular, sugerindo que, para cada sete pacientes com demência pré-senil, um apresentaria $\mathrm{DFT}^{2}$. Em outro estudo mais recente, a DFT foi considerada, juntamente com a DA, a forma de demência mais prevalente na população présenil estudada ${ }^{3}$. No Brasil, conduzimos um estudo que englobou pacientes com demência senil e pré-senil e encontramos $12,8 \%$ dos pacientes com esta forma de demência ${ }^{4}$ e, portanto, discordamos dos autores quando aventam a possibilidade de que sua prevalência de DFT espelha o que ocorre no restante do país. Uma explicação plausível para a baixa freqüência de DFT encontrada no estudo de Fujihara et al. se refere provavelmente ao local onde estes pacientes foram arregimentados. Como a DFT cursa predominantemente com alterações do comportamento (desinibição, apatia, hipersexualidade, entre outros), tais pacientes são encaminhados preferencialmente para serviços psiquiátricos e não neurológicos, como é o caso do serviço onde foi conduzida a pesquisa, qual seja, o Ambulatório de Neurologia Cognitiva do Hospital Santa Marcelina. Outro aspecto que pode levar ao subdiagnóstico de casos de DFT é a importância que se dá às alterações cognitivas para o diagnóstico de demência, em detrimento das alterações de comportamento. Os autores assinalaram na metodologia que os pacientes foram-Ihes encaminhados para avaliação de queixas cognitivas, o que pode criar um viés de seleção, não contemplando casos de DFT, cuja sintomatologia seja mais comportamental (a maior parte dos casos, diga-se).

\section{REFERÊNCIAS}

1. Fujihara S, Brucki SMD, Rocha MSG, Carvalho AA, Piccolo AC. Prevalence of presenile dementia in a terciary outpatient clinic. Arq Neuropsiquiatr 2004; 62: 592595.

2. Harvey RJ. Epidemiology of presenile dementia. In Hodges JR. Early-onset dementia. Oxford: Oxford University Press, 2001: 1-21.

3. Ratnavalli E, Brayne C, Dawson K, Hodges JR. The prevalence of frontotemporal dementia. Neurology 2002; 58: 1615-1621.

4. Caixeta L. Epidemiologia das diferentes formas de demência em Goiás. A rq Neuropsiquiatr 2003; 61 (Suppl 2): S 19.
Resposta dos Autores - O Dr. Caixeta salienta que a prevalência de demência frontotemporal (DFT) no Ambulatório de Cognição do Hospital Santa Marcelina está sub diagnosticada ${ }^{1}$. Não existem dados publicados em nosso país sobre a prevalência deste tipo de demência, que permitam tal afirmação. Devemos observar a diferença entre estudos populacionais, com grande variação entre os diferentes países, e ambulatoriais. De fato, em países europeus e na América do Norte, a prevalência de DFT varia de 12 a $20 \%$ das demências degenerativas. Considerando-se os casos de demências puramente degenerativas $(n=41)$, teremos uma prevalência de $17,1 \%$ de pacientes com DFT.

$\mathrm{O}$ autor refere que em seu estudo, tal prevalência foi $12,5 \% 2$. Porém, chamo atenção de que sua amostra além de ser limitada pelo número de dementes analisados $(n=$ 70), é heterogênea, pois provém de ambulatório público psiquiátrico, clínica privada e ambulatório de demências da Universidade Federal de Goiás. O viés de encaminhamento para estes locais parece-me maior do que para um ambulatório especializado em demências de uma região de abrangência de quase três milhões de pessoas, com 141 pacientes com demência pré-senil ${ }^{1}$. A demência de mais prevalência foi a demência vascular, $36,9 \%$, semelhante à encontrada em Hospital Psiquiátrico $(39,1 \%)^{3}$, o que nos parece provável em regiões pobres e com cuidados precários de fatores de risco para doenças vasculares. Atendo-nos aos dados disponíveis no Brasil (Tabela), claramente demonstram-se necessários trabalhos multicêntricos e de regiões diferentes do país para termos dados consistentes e definitivos sobre a prevalência de DFT, especialmente em estudos específicos em demência pré-senil, uma vez que os dados da Tabela mesclam pacientes de todas as idades atendidos nos respectivos ambulatórios.

Tabela. Porcentagem de DFT em artigos brasileiros.

\begin{tabular}{clcc}
\hline Referência & Local de coleta & $\mathrm{N}^{\circ}$ dementes & $\%$ de DFT \\
\hline 4 & HC FMRP & 186 & $3,23 \%$ \\
5 & HC UNICAMP & 261 & $3,4 \%$ \\
6 & HC FMUSP & 454 & $5,1 \%$ \\
\hline
\end{tabular}

\section{REFERÊNCIAS}

1. Fujihara S, BruckiSMD, Rocha MSG, Carvalho AA,Piccolo AC. Prevalence of presenile dementia in a tertiaty outpatient clinic. A rq Neuropsiquiatr 2004;62: 592-595.

2. Caixeta L. Epidemiologia das diferentes formas de demência em Goiás. Arq Neuropsiquiatr 2003;61(Supl 2):S19.

3. Canineu PR, Damasceno BP, Silva MC. Prevalência de demências na população de pacientes idosos (60 anos) internados no serviço de saúde Dr Cândido Ferreira da Prefeitura Municipal de Campinas. Arq Neuropsiquiatr 2003;61(supl 2):S21.

4. Vale FAC, Miranda SJC. Clinical and demographic features of patients with dementia attended in a tertiary outpatient clinic. Arq Neuropsiquiatr 2002;60:548552.

5. Silva DW, Damasceno BP, Silva MC. Demência na população de pacientes do Hospital das Clínicas da UNICAMP. Arq Neuropsiquiatr 2002;60:996-999.

6. Takada LT, Caramelli P, Radanovic M, et al. Prevalence of potentially reversible dementias in a dementia outpatient clinic of a tertiary university-affiliated hospital in Brazil. Arq

7. Neuropsiquiatr 2003;61:925-929.

Sonia Maria Dozzi Brucki 